
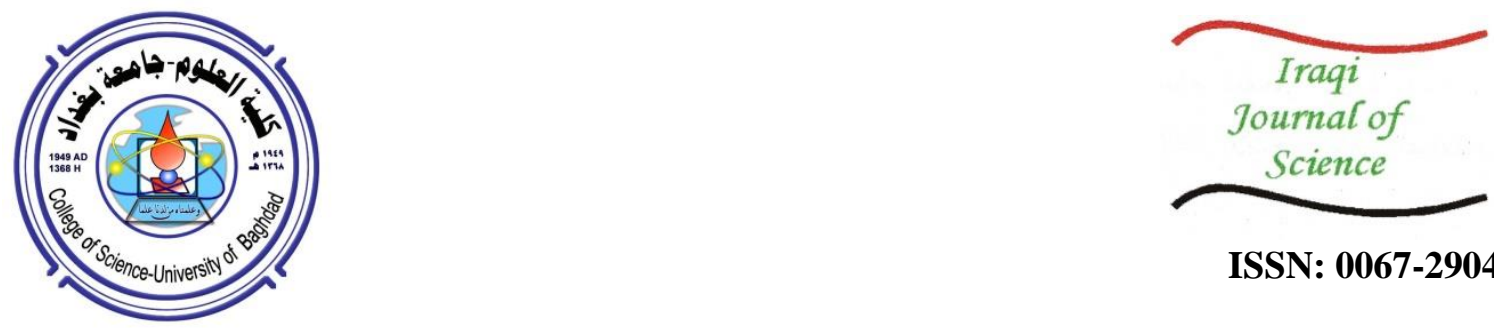

ISSN: 0067-2904

\title{
Textural Analysis of Liver Tumor using Watershed Segmentation based on Statistical and Geometrical features
}

\author{
Maysaa R. Naeemah \\ Physics Department, College of Science for Women, University of Baghdad, Baghdad, Iraq
}

\begin{abstract}
The liver diseases can define as the tumor or disorder that can affect the liver and causes deformation in its shape. The early detection and diagnose of the tumor using CT medical images, helps the detector to specify the tumor perfectly. This search aims to detect and classify the liver tumor depending on the use of a computer (image processing and textural analysis) helps in getting an accurate diagnosis. The methods which are used in this search depend on creating a binary mask used to separate the liver from the origins of the other in the CT images. The threshold has been used as an early segmentation. A Process, the watershed process is used as a classification technique to isolate the tumor which is cancer and cyst.

The test images are taking five for cancer case and five for the cyst, the geometrical and statistical features are calculated for both cases to identify the identity for each case. The statistical features are obtained from the gray level co-occurrence matrix which is contrast, homogeneity, correlation and energy. The geometrical features are the area of the tumor, the circumference and irregularity. The irregularity tends to lose one of the cyst cases than for cancer because of its shape in more regular than cancer.
\end{abstract}

Keywords: CT images, geometrical features, statistical features, tumor liver, cooccurrence matrix.

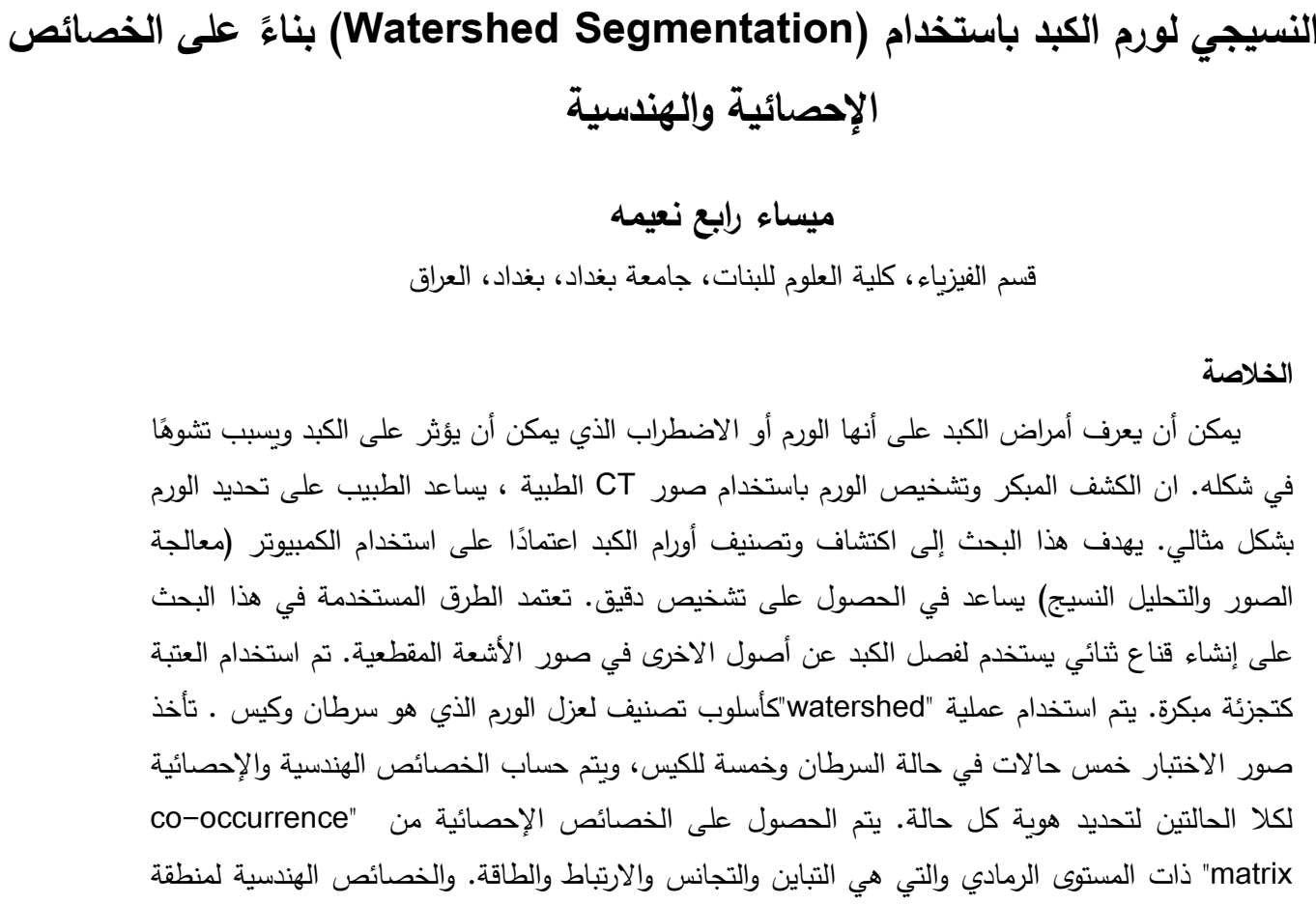




$$
\text { لأن شكله أكثر انتظامًا من السرطان. الانتام الذي تميل فيه المخالفة إلى فقدان أحدها في حالة الكيس مقارنة بالسرطان ، }
$$

\section{Introduction}

The medical image processing is widely used for helping the doctor in the liver tumor diagnosis. The medical image processing plays an important tool in producing a lot of information about the human body inside - the CT computed tomography give a lot of information about the liver texture. Image segmentation is a process of partitioning an image into a set of non-overlapping regions. It is the division of an image into regions or categories, which correspond to different objects or parts of objects. The goal of segmentation is to simplify and change the representation of an image into something that is more meaningful and easier to analyze. It is typically used to locate objects and boundaries (lines, curves, etc.) in an image. Thresholding is one of the simplest and most widely used approaches in image segmentation. Most of the existing thresholding methods are bi-level, which use two levels to categorize the image into background and object segments. It can extract the object from the background by grouping the intensity according to the threshold [1]. The watershed segmentation is a process of image classification; it is first used by Beucher [2]. A watershed transform is a basinlike landform plain by highpoints and ridgelines that fall into lower elevation and waterway valleyhaveand it is used to perform some morphological operations. It is a segmentation method in arithmetic morphology. The watershed transform is a morphological gradient-based segmentation technique. The watershed classification is used to locate the tumor out of the rest CT images to make the region of interest clearly and it became easy to obtain the geometrical and textural features which can be in calculated from the second order gray level co-occurrence matrix which is homogeneity, contrast, energy and correlation [3].

\section{Methodology}

The CT image which has been taken must be converted to HSV system to separate the RGB layer to make the classification more specific. The HSV stands for the Hue, Saturation and Value and RGB is which R stands for red, G represents the green and B is the blue. The value represents the intensity of a color, which is decoupled from the color information in the represented image. The hue and saturation components are intimately related to the way the human eye perceives. The transformation equations for RGB to HSV color model conversion are given [4]:

$$
\begin{aligned}
& V=\max (R, G, B) \ldots \ldots \\
& S=\frac{V-\min (R, G, B)}{V} \ldots \\
& H=\frac{G-B}{6 S} \quad, \text { if } V=R \ldots
\end{aligned}
$$

Where $\mathrm{H}$ is the hue, the $\mathrm{S}$ is the saturation and $\mathrm{V}$ is the value.

Two cases of diseases are used in this search five for cancer and five for cysts. Ten CT image are taken to separate the region of interest from the whole image, a binary mask is used. The threshold is one of the simplest image segmentation techniques. It is selected according to the purpose for which it is applied [5]. It is a method for segmentation gray levels images; the gray scale image is converted to a binary, black and white image. [6]. for single thresholding, The $\mathrm{T}$ is the threshold value given by the following equation [7]:

$$
g(x, y)=\left\{\begin{array}{lll}
1 & \text { if } & f(x, y)>T \\
0 & \text { if } & f(x, y) \leq T
\end{array}\right.
$$

The HSV (Hus Saturation and value) are used to color the image the watershed is used to segment the image in order to separate the tumor from the healthy tissue. Using the morphological (open and close) to select perfectly the study region, often the region has been selected, the statistical features which are the second order statistical features are homogeneity, contrast correlation and energy. The second feature is the geometrical features which are the area, circumference and irregularity. The watershed technique is the most commonly used in several fields of image processing, including medical image [8]. It is a useful tool in image segmentation. In which watershed lines can effectively divide individual catchment basins in a gradient image and generate closed contours for each region 
in the original image. The methodologies of image segmentation which based upon watershed transform have been developed [9].The watershed segmentation process follows the steps.

1-The segmented parts are over together when the local minima of the gradient have been marked. This process leads to produce a problem. To get rid of this a "Meyer and Beucher" produced a method called the "marker controlled segmentation" [10].

2- Each position must be marked either by the user or by the morphological operator when the morphological properties are used the objects with in the images has its own properties which mark the objects, this is called "object markers" the "background markers" in which there is no pixel related to the objects.

The area of the markers is obtained by obtaining the "specific contours" this can be getting by obtaining the gradient image. The gradient image is an essential process in the "watershed segmentation process" when process refers to the homogeneity, the distance between the pixel is need when the segment refers to the shape of the objects,i.e., the two processes works together. The "gradient" and the "distance" it's called the "morphological operation". The open and close are process belongs to the morphological operation. The open process is an erosion process then dilation, the erosion is an operator which break the thin edges between the nearest object is based on removing the noise and the outer layer from the larger objects, as a result the size of the holes within the object increase while, the dilation operator aims to fill the holes and add layers to the objects, the close function is a dilation process followed by the erosion.

\section{The statistical features:}

The co-occurrence matrices are one of the methods used to extract the features which are constructed by considering that every pixel has eight neighbors (horizontally, vertically and diagonally at 45 degrees). It also assumed that the matrix of relative frequencies of gray levels co-occurrence can specify the texture-context information. Some of the texture measures can be obtained from these matrices, (like homogeneity, correlation, energy, and contrast). The texture is specified by the matrix of relative frequencies of co-occurrence $p(i, j)$, which indicate the number of times that each two neighboring pixels of an image, separated by a distance (d), will have gray tone (i) for one pixel and (j) gray tone for the other pixel. Such matrices of gray tone spatial dependence frequencies are the functions of the angular relationship between the neighboring pixels, as well as a function of the distance between them [11]. The co-occurrence matrices are based on the repeated occurrence of the gray level configuration in the considered texture. This configuration varies rapidly in fine textures, slowly more in coarse textures.

The most important texture features which are used in this search are [12]:

Contrast: It determines the intensity difference between a pixel and its neighborhood; it can be represented by the following equation:

$$
\text { contrast }=\sum_{i=0}^{G-1} \sum_{j=0}^{G-1}(i-j)^{2} P(i, j)
$$

Where $\mathrm{G}=256$.

Correlation: It measures the pixel correlation and how it is correlated with its neighborhood pixels. Its value lies between $(-1$ and +1$)$, when the value is $(-1)$ it is negatively correlated and when it is $(+1)$ it's positively correlated. It is defined as:

$$
\text { correlation }=\sum_{i=1}^{G-1} \sum_{j=1}^{G-1} \frac{P(i, j)\left(i-\mu_{x}\right)\left(j-\mu_{y}\right)}{\sigma_{x} \sigma_{y}}
$$

Where $\mu_{x}, \mu_{y}$ : Mean value in the $\mathrm{x}$ and $\mathrm{y}$ direction. $\sigma_{x}, \sigma_{y}:$ Standard deviation in both direction $(\mathrm{x}, \mathrm{y})$.

Homogeneity: It measures the purity of the image texture, it can be represented by the following equation:

$$
\text { Homogeneity }=\sum_{i, j=1}^{G-1} \frac{P(i, j)}{1+(i-j)^{2}}
$$

Energy: It is the sum of squared elements. It ranges from ( 0 to 1$)$, it is inversely proportional to entropy. It can be represented by the following equation:

$$
\text { energy }=\sum_{i, j=1}^{G-1} P(i, j)^{2}
$$

Geometrical Features: The geometrical features can be described by determining the area, perimeter and irregularity index. The area is finding by summing all the white pixels in the image matrix which has one value, while the background which has zero value is ignored. The irregularity index is computed by the following equation. 


$$
\begin{aligned}
& \text { Area }=\sum \text { White Area } \\
& I=\frac{4 \pi A}{P^{2}}
\end{aligned}
$$

When $\mathrm{p}$ is representing the perimeter of the tumor and $\mathrm{A}$ is the area of the tumor in units called pixels. The irregularity has a value equal to 1 only for circular shape and it is $<1$ for any other shape [13].

\section{The study cases}

Cysts cases: - The liver cyst is one of the liver tumors it has a regular shape comparing with other tumor and it considers as a benign tumor of liver cell, large one cause pain and bleeding and it must be removed. Figure-(1, 2, 3, 4, and 5) shows the cysts study cases. The liver cyst can be separated from the other healthy part by using the watershed segmentation and by calculating the geometrical and statistical textural features. Table-1 represented the textural features, while Table- 3 the geometrical features.

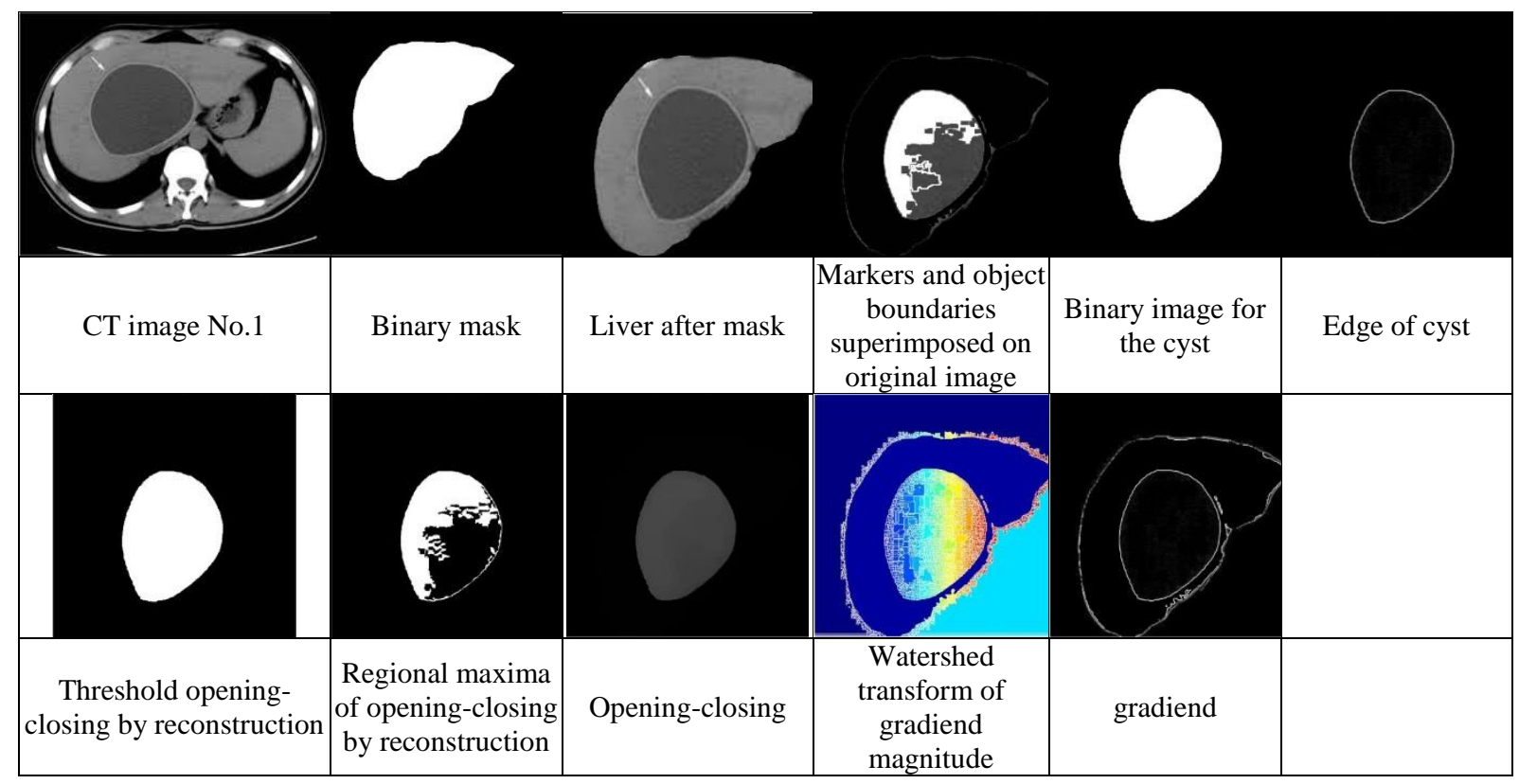

Figure 1- the watershed segmentation with cyst case for Image No.1

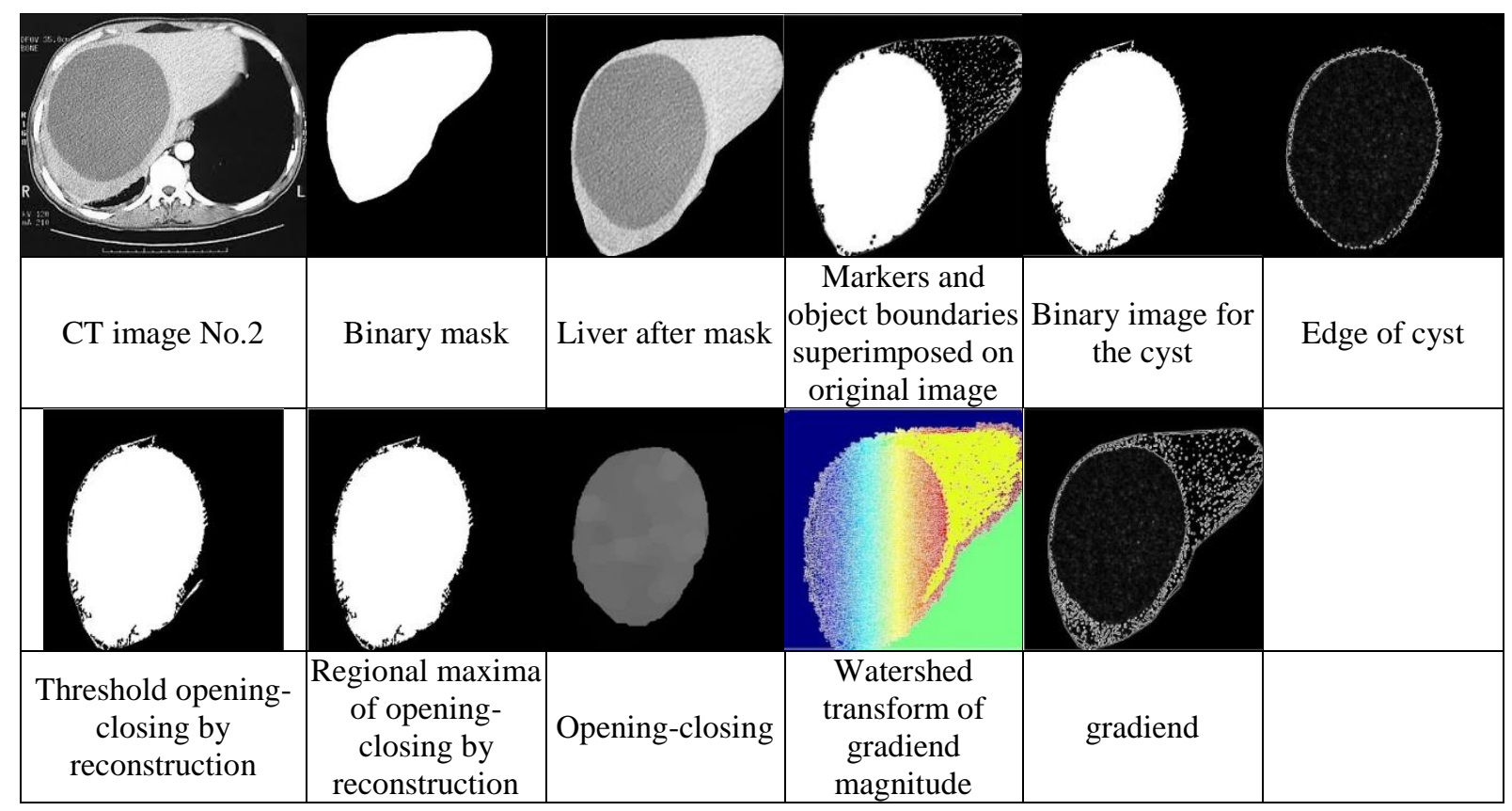

Figure 2-the watershed segmentation with cyst case for Image No.2 


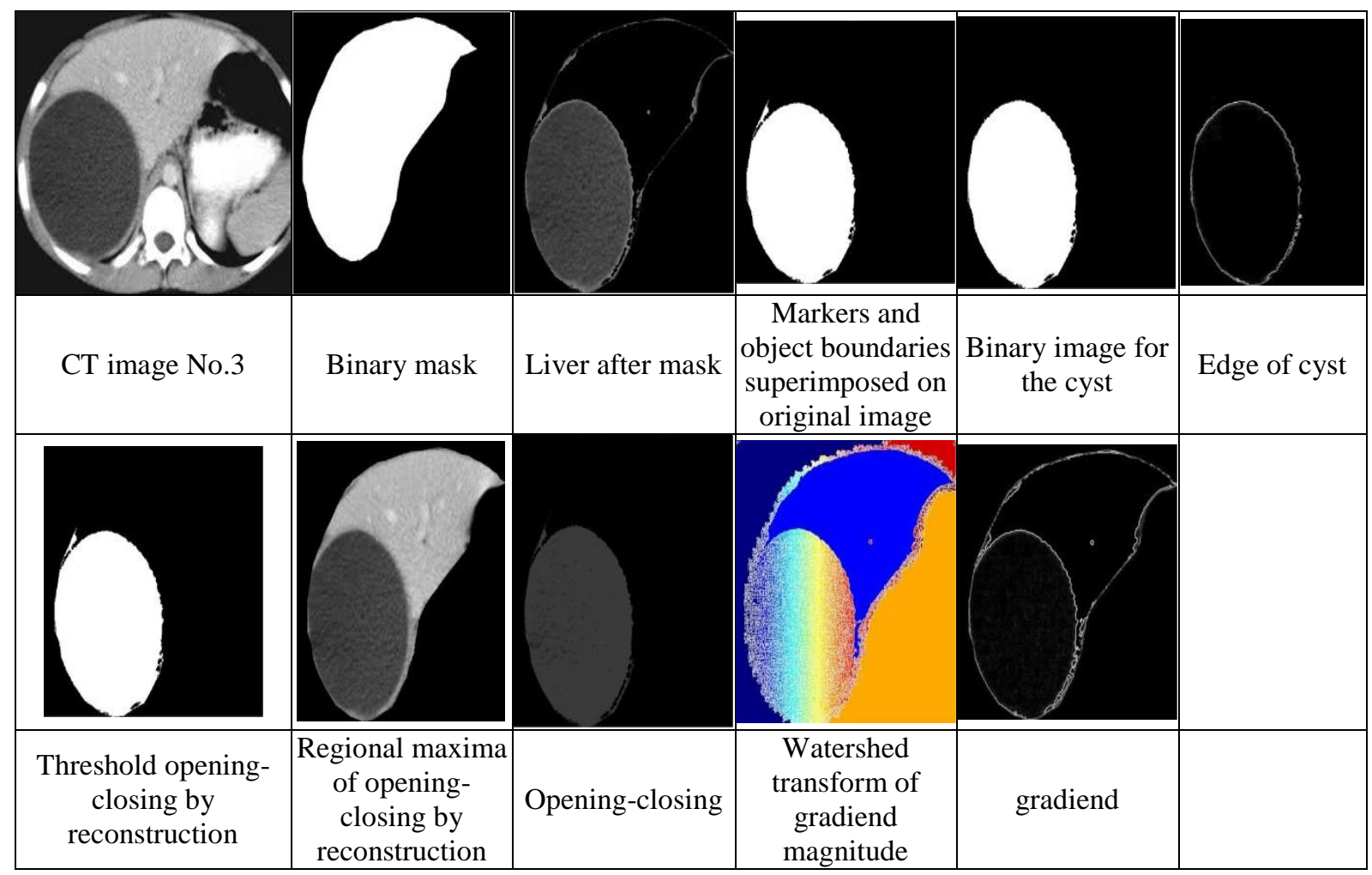

Figure 3-the watershed segmentation with cyst case for Image No.3

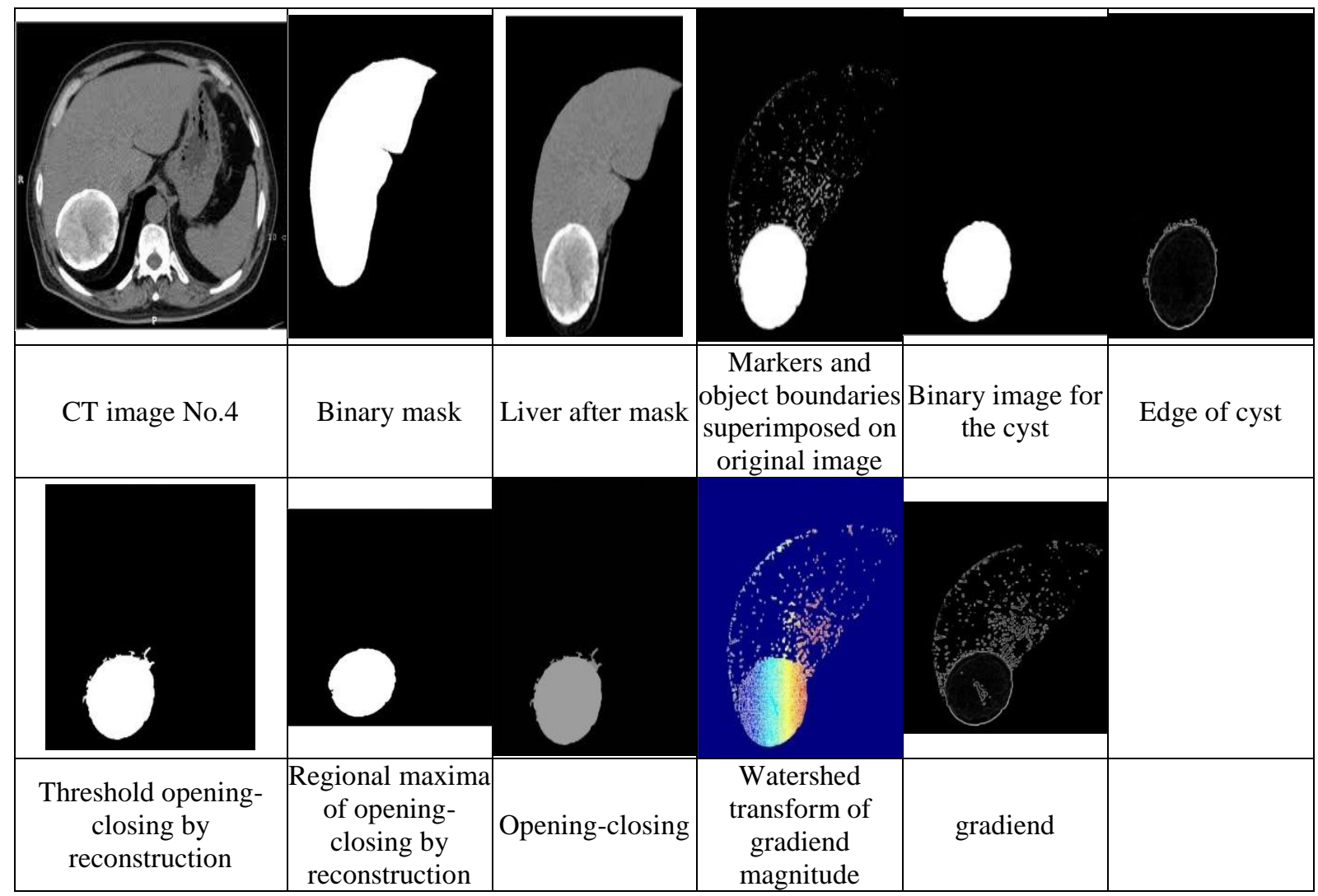

Figure 4-the watershed segmentation with cyst case for Image No.4 


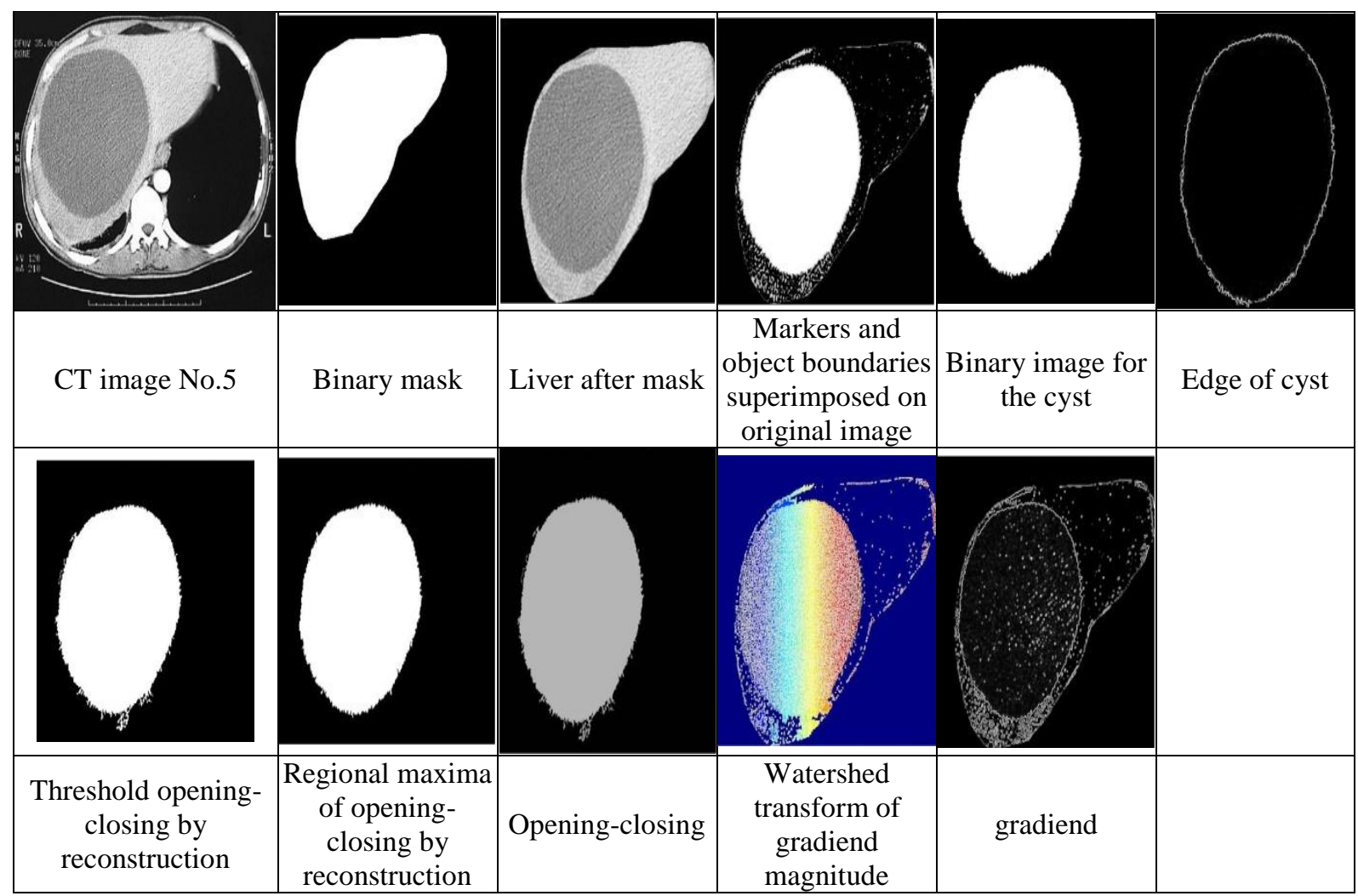

Figure 5-the watershed segmentation with cyst case for Image No.5

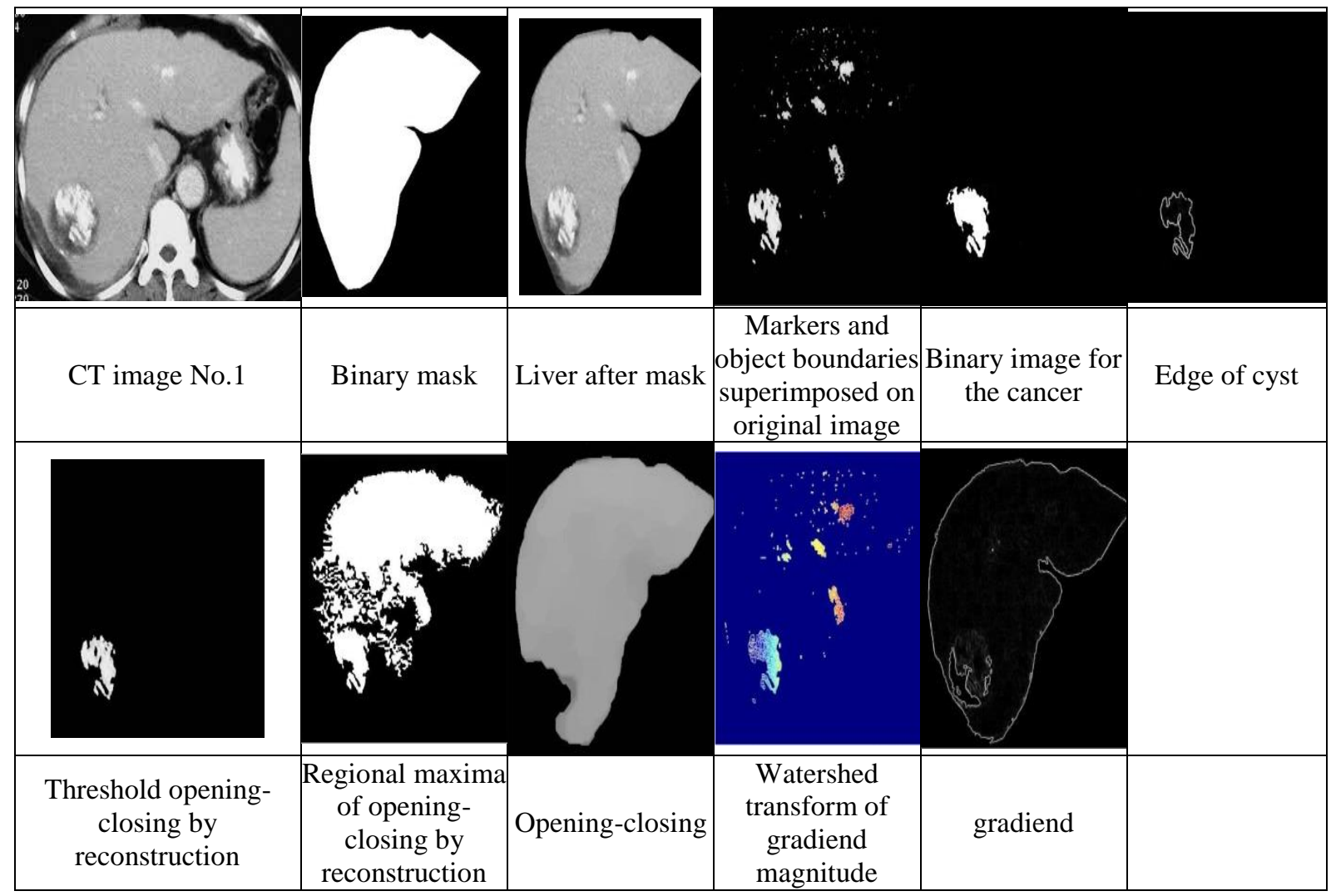

Figure 6- the watershed segmentation with cancer case for Image No.1 


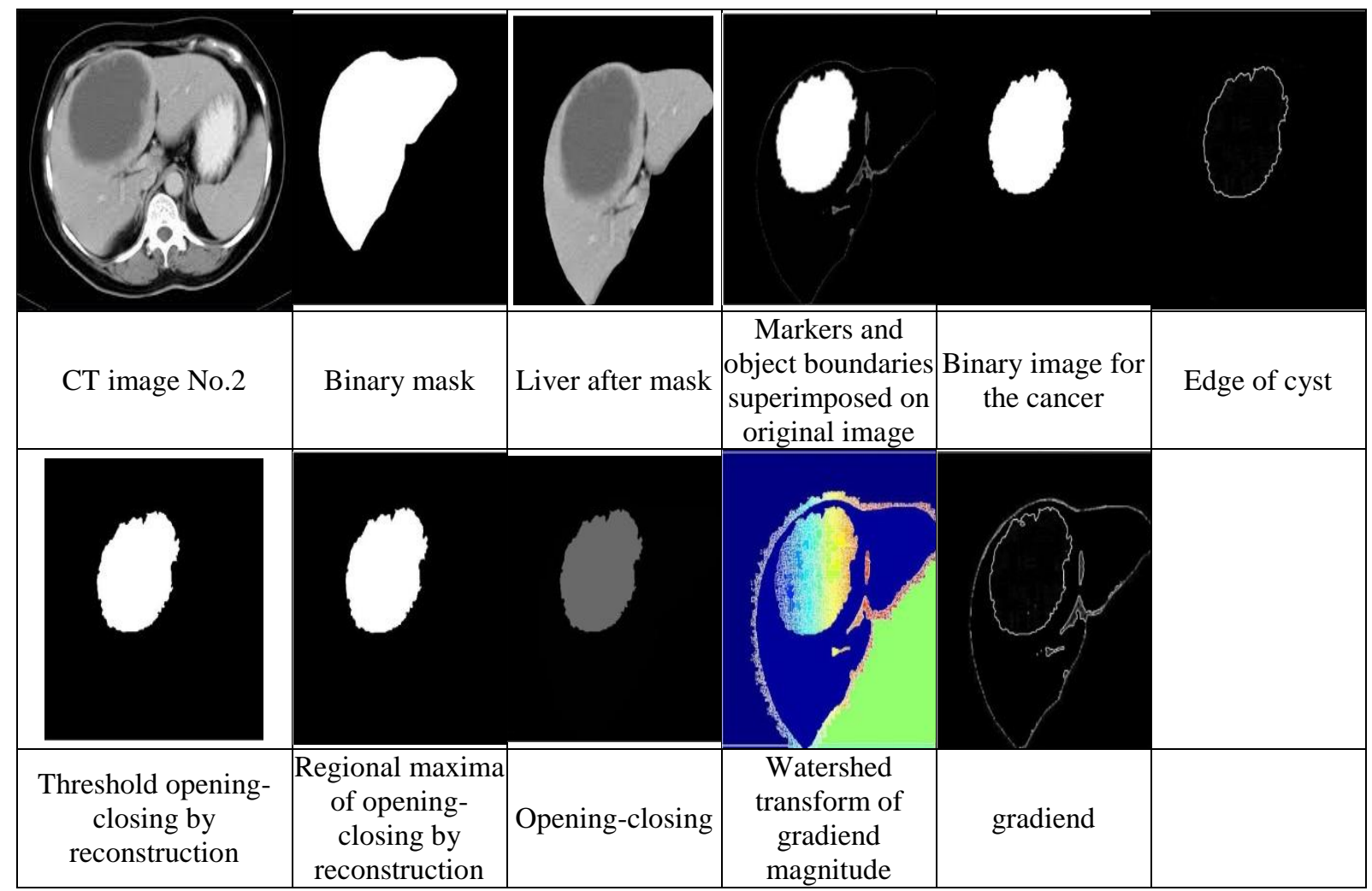

Figure 7-the watershed segmentation with cancer case for Image No.2

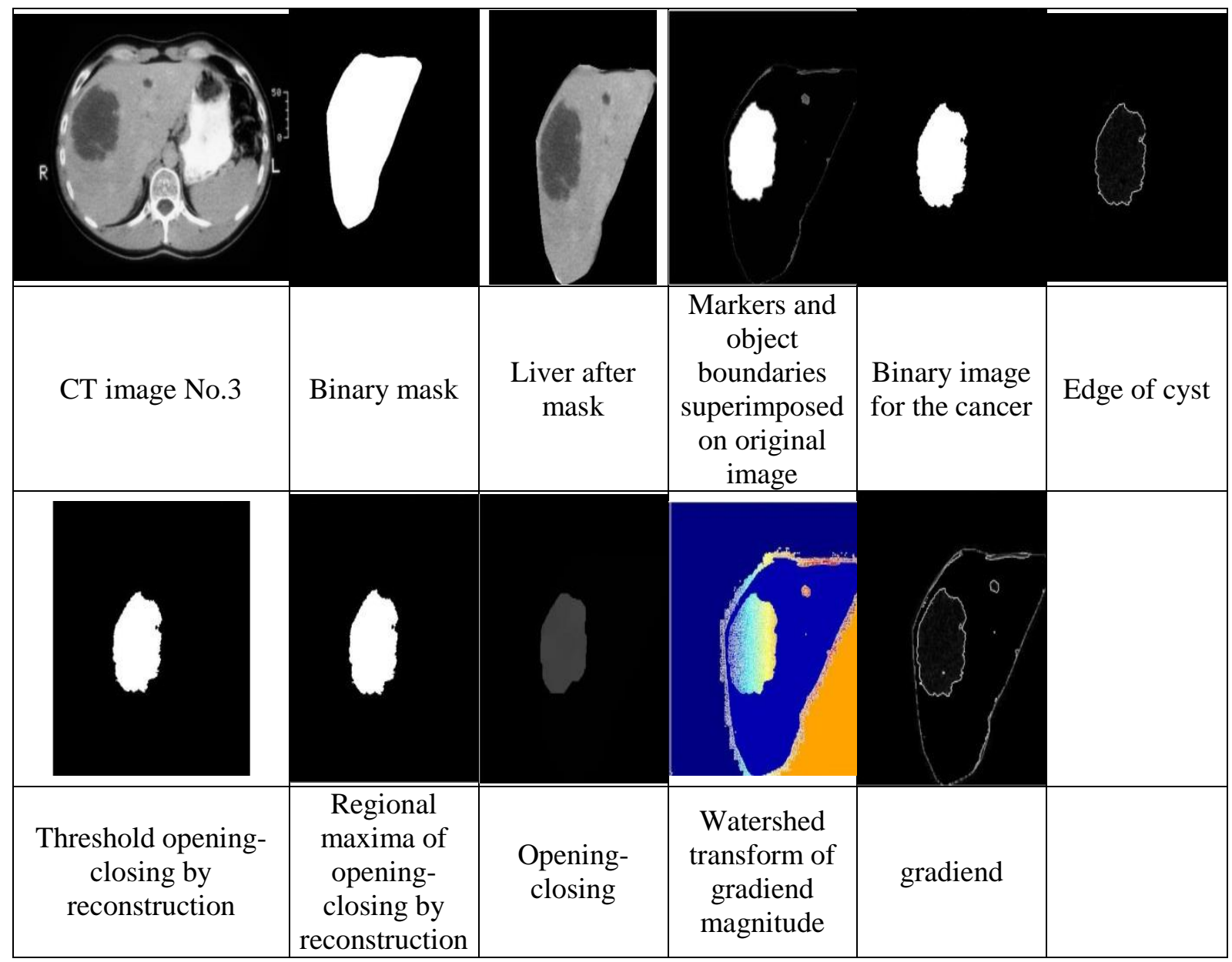

Figure 8-the watershed segmentation with cancer case for Image No.3 


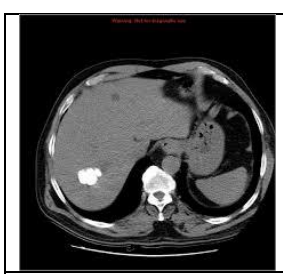

CT image No.4

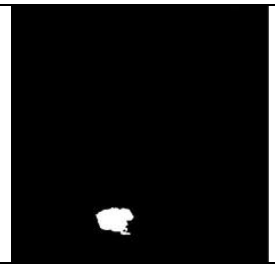

Threshold openingclosing by reconstruction

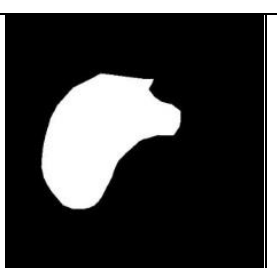

Binary mask

Liver after mask

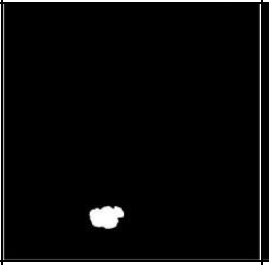

Regional maxima of opening-

closing by

reconstruction

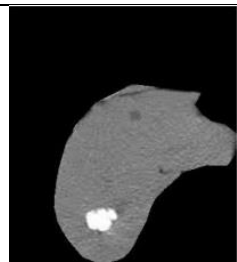

Opening-closing

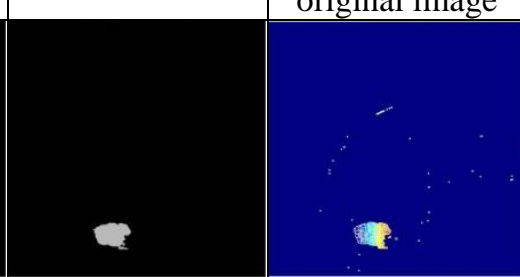

Watershed transform of gradiend magnitude

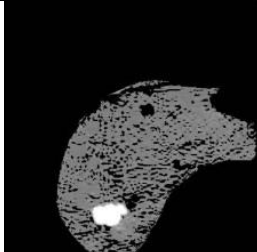

Markers and object boundaries superimposed on original image

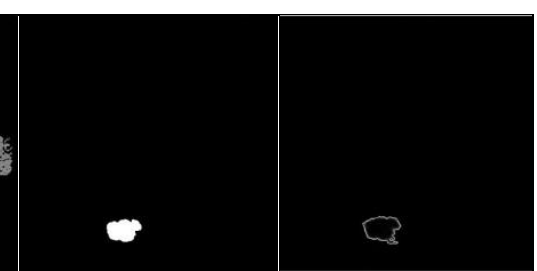

Binary image for the cancer

Edge of cyst

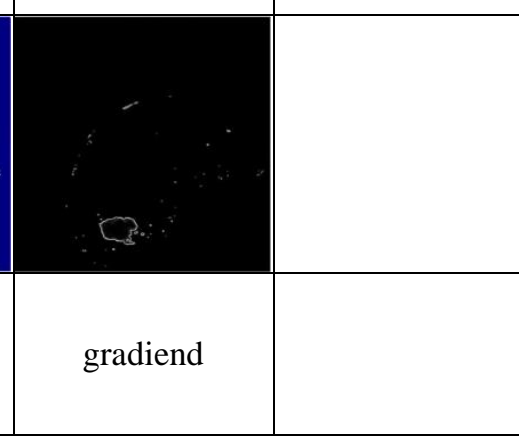

Figure 9-the watershed segmentation with cancer case for Image No.4

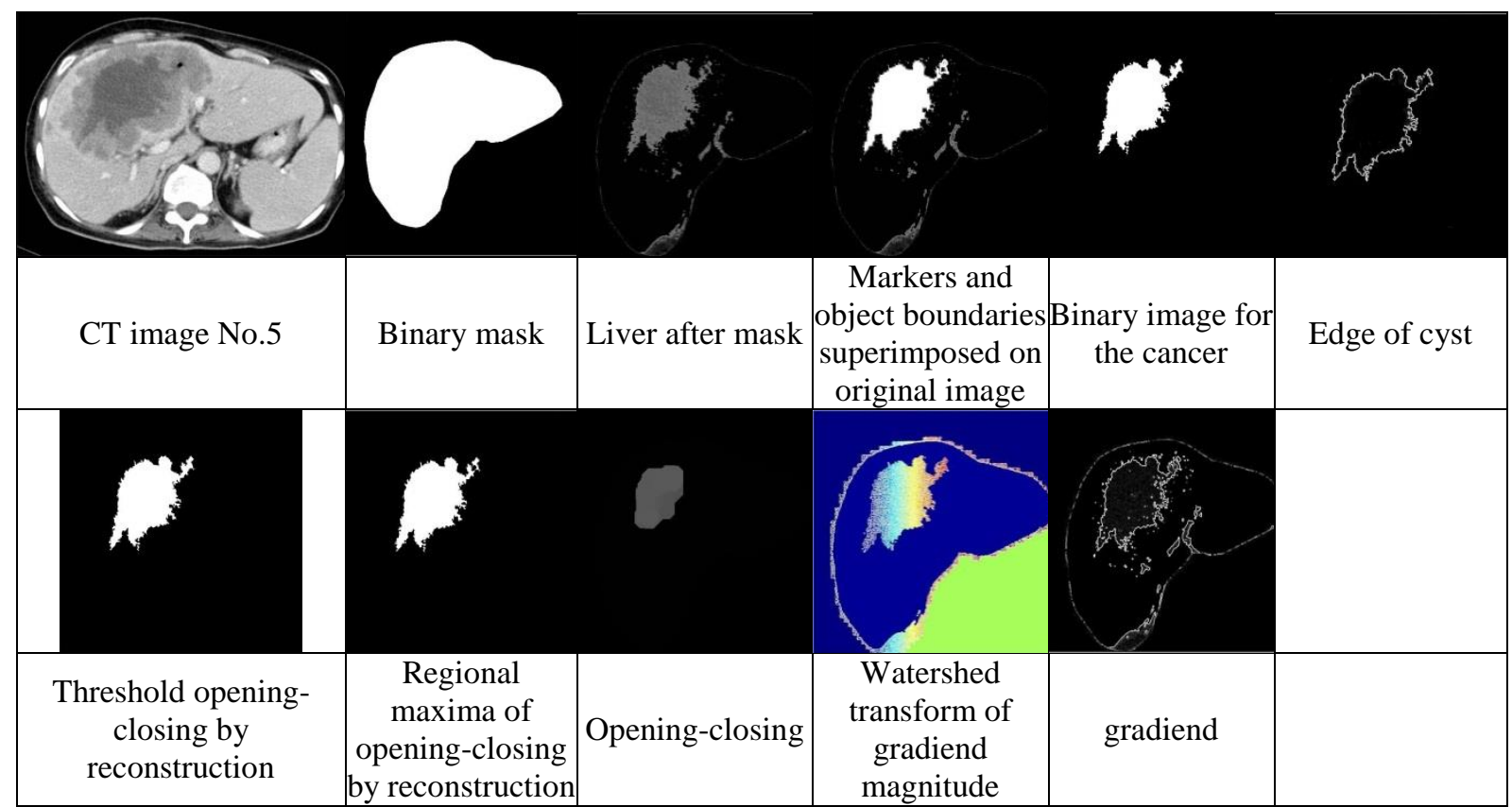

Figure 10-the watershed segmentation with cancer case for Image No.5

Table 1- the statistical features to liver the cysts case

\begin{tabular}{|c|c|c|c|c|}
\hline Image No. & Contrast & Correlation & Energy & Homogeneity \\
\hline 1 & 0.01355 & 0.9876 & 0.71265 & 0.99655 \\
\hline 2 & 0.0399 & 0.99 & 0.55355 & 0.99445 \\
\hline 3 & 0.0544 & 0.89465 & 0.60565 & 0.9966 \\
\hline 4 & 0.10805 & 0.9587 & 0.8368 & 0.9933 \\
\hline 5 & 0.19215 & 0.9825 & 0.5407 & 0.9878 \\
\hline
\end{tabular}


Table 2-the statistical features to liver the cancer case

\begin{tabular}{|c|c|c|c|c|}
\hline Image No & Contrast & Correlation & Energy & Homogeneity \\
\hline 1 & 0.1892 & 0.93015 & 0.95675 & 0.99365 \\
\hline 2 & 0.076 & 0.9626 & 0.77755 & 0.9952 \\
\hline 3 & 0.0118 & 0.977 & 0.86375 & 0.9963 \\
\hline 4 & 0.0739 & 0.88805 & 0.9731 & 0.99745 \\
\hline 5 & 0.03125 & 0.94295 & 0.85995 & 0.9938 \\
\hline
\end{tabular}

Table 3-the geometrical features to liver for the cysts case

\begin{tabular}{|c|c|c|c|}
\hline Image No & Area & Circumference & Irregularity \\
\hline 1 & 3766 & 481 & 0.20444 \\
\hline 2 & 12369 & 3533 & 0.01244 \\
\hline 3 & 8072 & 716 & 0.19776 \\
\hline 4 & 2638 & 675 & 0.72720504 \\
\hline 5 & 9879 & 1962 & 0.032233288 \\
\hline
\end{tabular}

Cancer cases: The liver cancer is a mass growth in the liver itself and started in the cells of the blood vessels and grew into multiple tumors. Figures-(6, 7, 8, 9, 10,11) represented the cancer case. The textural statistical features and geometrical features are calculated (Table-2)) listed textural features while Table-4 represented the geometrical features.

Table 4- the geometrical features to liver the cancer case

\begin{tabular}{|c|c|c|c|}
\hline Image No & Area & Circumference & Irregularity \\
\hline 1 & 17207 & 3801 & 0.014958 \\
\hline 2 & 37536 & 1791 & 0.14 \\
\hline 3 & 27242 & 3244 & 0.03251374 \\
\hline 4 & 9256 & 1307 & 0.06855277 \\
\hline 5 & 3235 & 4265 & 0.022337742 \\
\hline
\end{tabular}

Table 5-the average value for the statistical features for the Cyst and cancer case

\begin{tabular}{|c|c|c|c|c|}
\hline case & Contrast & Correlation & Energy & Homogeneity \\
\hline cyst & 0.081 & 0.962 & 0.6498 & 0.993 \\
\hline cancer & 0.076 & 0.9401 & 0.886 & 0.995 \\
\hline
\end{tabular}

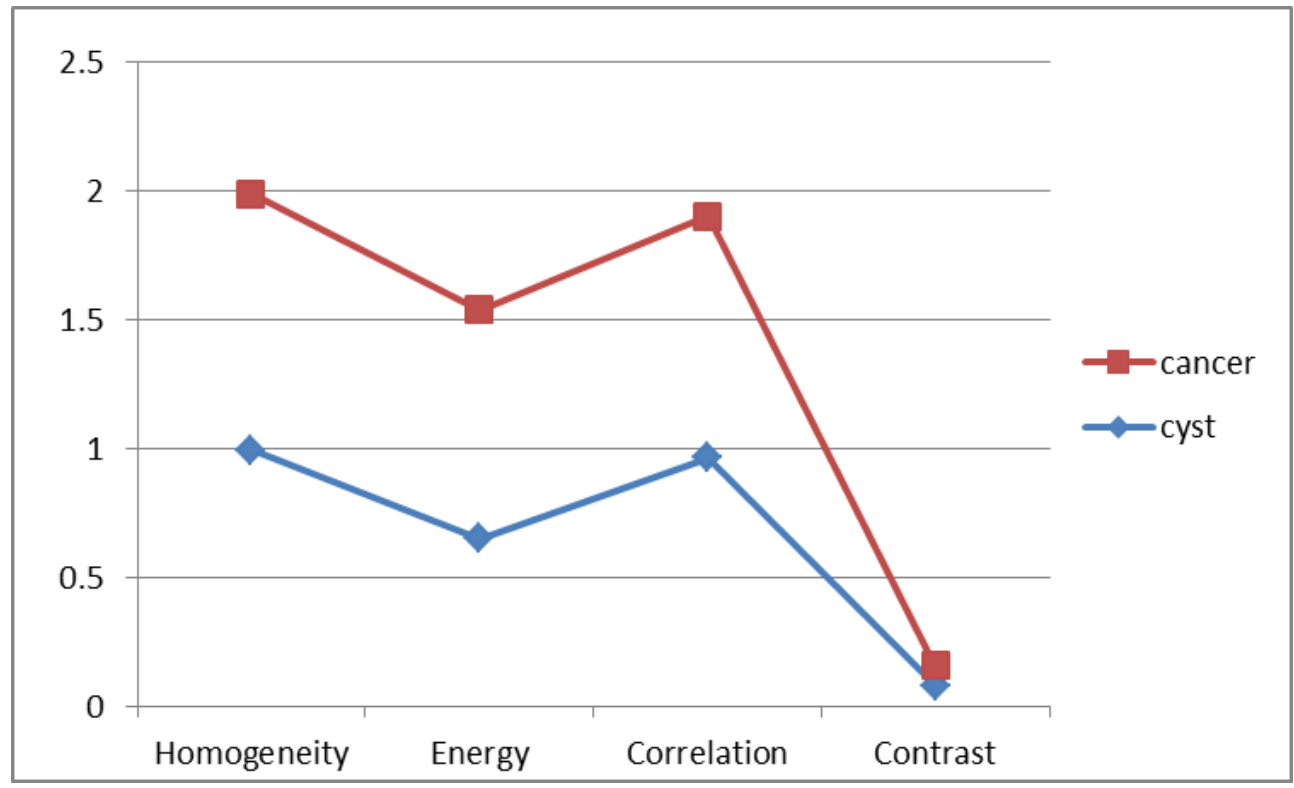

Figure 11-The statistical features for cyst, and cancer case. 
Table 6-the average value for the geometrical features for the Cyst and cancer case

\begin{tabular}{|c|c|c|c|}
\hline case & Area & Circumference & Irregularity \\
\hline cancer & 69976 & 14408 & 0.055 \\
\hline cyst & 36724 & 7367 & 0.235 \\
\hline
\end{tabular}

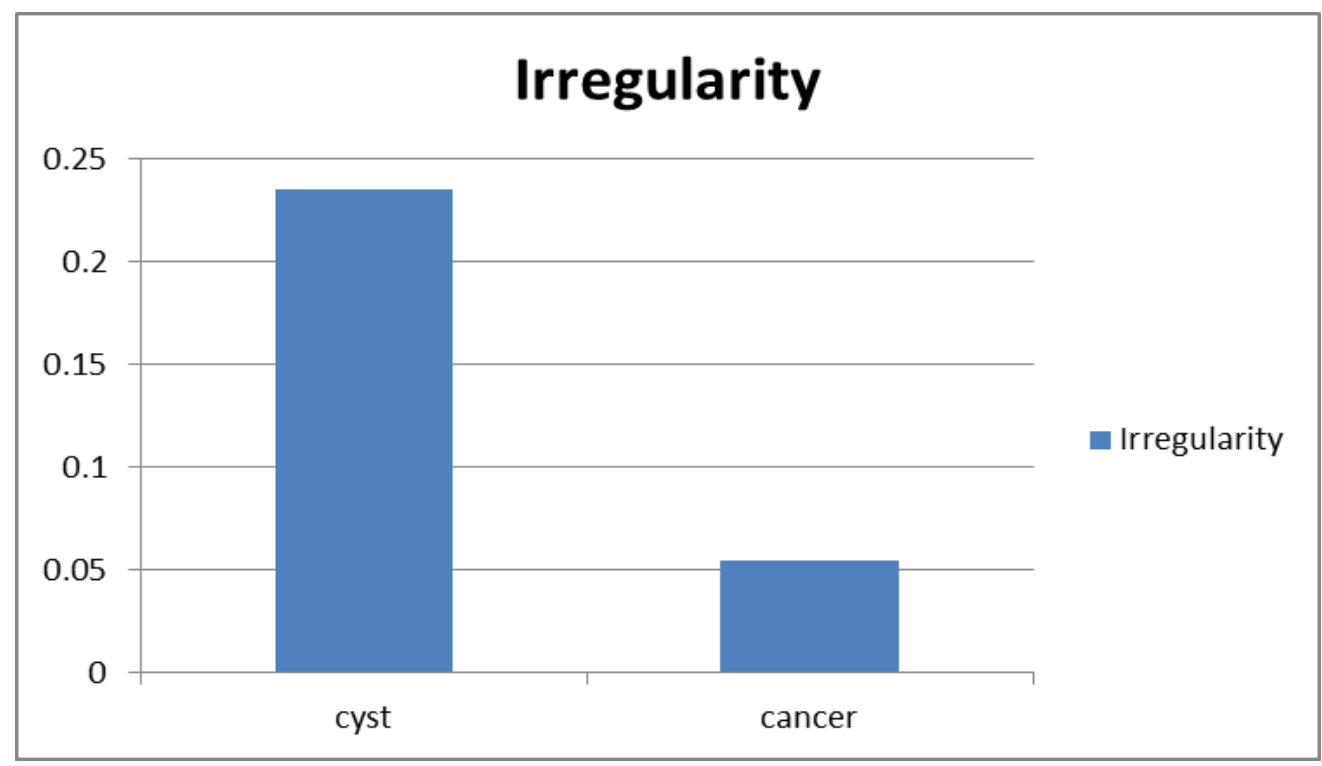

Figure 12-the geometrical features for cyst, and cancer case.

\section{Discussion}

The statistical features which give inclination about the texture properties, are calculated to distinguish between the two type tumor, the correlation features which deal with the neighbor pixel as the correlation has high value, there is no detect in the tissue is maximum value is +1 when the tissue is highly correlated while the value -1 is when there is no relation between them.

The contrast measures the number of variations in the images when the contrast is high this means higher variations are in the image.

The energy value measures the textural uniformity, it detects the disorders in the texture. When there is no disorder in the texture value is (one). The homogeneity is a measure of party of the texture its value is large for small gray lever distribution (i.e.) the element in the image is the same. Table (5) indicated the result for the average value for the five cases for both cancer and cyst, Table- 6 represented the average value for the geometrical features in each cyst and cancer.

\section{Conclusion}

From the result shown in the Tables- $(5,6)$ the cyst contrast is higher than that for cancer so is the correlation this means that the texture of cyst has a number of a gray level larger than that for cancer and its texture is less purity than that for cancer. This is clearly seen from the homogeneity and energy for which energy and homogeneity are less than that for cancer, the cancer texture has less number of the gray level so, it correlation value is less compared with the cyst. Its value of energy is high than that for cyst, the cancer texture has homogenous texture compare with that of the cyst. From the geometric features the shape of cyst is more irregular than that for cancer this can be clearly seen from the Figure-12 and Table- 6 of each of cancer and cysts. As a result, the geometrical features give good information about the liver tumor compared with the statistical features, and the energy feature which is a statistical feature is the best features for considering the liver diagnoses. 


\section{References}

1. Sohi, N., Kaur, L. and Gupta, S. 2012. Performance Improvement of Fuzzy C-Mean Algorithm for Tumor Extraction in MR Brain Images. International Journal of Computer Applications (IJCA), 59(5): 40-45.

2. Kaur, A. and Verma, A. 2013. The Marker-Based Watershed Segmentation- A Review. International Journal of Engineering and Innovative Technology (IJEIT). 3(3): 171-174.

3. Napoleon, J., Santhoshi, R. and Shameena, A. 2013. Verdict of Objects in Medical Images Using Marker-Controlled Watershed Image Segmentation . International Journal of Computer Applications (IJCA), 1: 1-4.

4. Ali, A.H., Jaafer, Z., Jasim, A. and Naeemah, M.R. 2017. Classification of Lung Diseases Based on K-mean Clustering. Journal of Pharmacy and Biological Sciences (IOSR-JPBS).12(5): 24-31.

5. Kaur, J., Agrawal, S. and Vig, R. 2012. A Comparative Analysis of Thresholding and Edge Detection Segmentation Techniques. International Journal of Computer Applications(IJCA). 39(15): 29-34.

6. McAndrew, A. 2004. An Introduction to Digital Image Processing with MATLAB. Boston, Thomson.

7. Huang, Z.K. and Chau, K.W. 2008. A New Image Thresholding Method Based on Gaussian Mixture Model. Applied Mathematics and Computation. University Hong Kong China. 205(2): 899-907.

8. Kaur, A. and Aayushi. 2014. Image Segmentation Using Watershed Transform. ". International Journal of Soft Computing and Engineering (IJSCE). University Putiala, India. 14(1): 2231-2307.

9. Vashist, P. and Hema, K. 2013. Watershed Transform on Image Segmentation and Data Classification. International Journal of Science and Technology (IJST) . 2(1): 112-121.

10. Ali, A.H. 2017. Studying the Kidney Textural Using Statistical Features and Local Binary Pattern. Journal of Al-Nahrain University. 20: 64-76.

11. Abod, Z.J. 2017. Detection and Classification of Lung Disease for Chest CT Images. M.A thesis, Department of Physics, College of Science for Women, Baghdad University, Iraq.

12. Aggarwal, N., and Agrawal, R.K. 2012. First and Second Order Statistics Features for Classification of Magnetic Resonance Brain Images. Journal of Signal and Information Processing (JSIP). 3(2): 146-153.

13. Mazhir, S.A., Ali, A.H. and Hadi, E.M. 2015. Diagnosis of liver Tumor from CT Images Using Unsupervised Classification with Geometrical and Statistical features. International Journal of Advanced Research in Computer vvvvScience and Software Engineering (IJACSSE). 5(3): 2835 . 